\title{
Processing of unattended visual information
}

\author{
GEORGE WOLFORD \\ Dartmouth College, Hanover, New Hampshire 03755 \\ and \\ FRED MORRISON \\ University of Minnesota, Minneapolis, Minnesota 55455
}

\begin{abstract}
A visual analog of auditory selective attention paradigms is described. Using that analog, we examined the amount of processing required to monitor unattended information. With the materials used, there was no evidence that any processing capacity was required to monitor the unattended information. Memory for the unattended information and the recognition of one's name were examined to provide additional evidence concerning the fate of the unattended information.
\end{abstract}

When confronted with two simultaneous messages, we generally attend to only one of the messages. There is evidence, however, that the unattended message is processed by the listener to some extent. Presented with auditory information, listeners are certainly aware of the gross physical characteristics of the unattended message (Broadbent, 1958). They also notice the presence of their name on the unattended message some percentage of the time (Moray, 1959). Indeed, a growing body of evidence suggests that material on the unattended ear may be processed to a semantic level. Treisman (1960) showed that when the theme of the attended message was switched to the unattended ear, subjects followed the message to that ear for some number of words. She also demonstrated that subjects recognize that the message on the unattended ear is identical to that on the attended ear under appropriate values of lag and lead (Treisman, 1964). Lewis (1970) found that shadowing latency was substantially increased when synonyms were presented to the unattended ear. Treisman, Squire, and Green (1974) verified the synonym effect but found that it was limited to the early part of a list. Additional evidence for the semantic processing of unattended input comes from an experiment by Lackner and Garrett (1972) and a series of similar experiments by MacKay (1973). Subjects were simultaneously presented ambiguous sentences to the attended ear and information to the unattended ear that would disambiguate the sentences. Subjects were then asked to paraphrase the attended sentences or to choose from a set of alternatives the sentence closest in meaning to the sentence presented. The unattended information significantly

This research was supported, in part, by NIMH Grant MH 33179, awarded to the first author, and an NIMH Research Scientist Development Award MH 0026, awarded to the second author. Requests for reprints should be sent to George Wolford, Psychology Department, Gerry Hall, Dartmouth College, Hanover, New Hampshire 03755. affected the direction in which the attended sentences were disambiguated, even though subjects reported no awareness or memory of the unattended information. Newstead and Dennis (1979) presented evidence that MacKay's results may be limited to certain conditions, such as when sufficient time is allowed between sentences on the attended ear.

Much of the work on selective attention has used the auditory modality. In addition to the methodological advantages of auditory paradigms (Broadbent, 1958), audition can be characterized by two distinct and obvious channels (Kahneman, 1973). In the visual modality, the notion of distinct channels is less appealing; also, in vision, there is a good physical mechanism for selective attention, namely, looking at or away from some location. It is possible that selective attention at the cognitive level evolved for auditory input and is specific to auditory input. Neisser (Note 1) did develop a visual analog to the auditory paradigms by constructing paragraphs in which every other line was printed in red and subjects were asked to ignore the red lines. Using that paradigm, Neisser was able to replicate several phenomena found with auditory paradigms, including Moray's (1959) name effect. One drawback of Neisser's paradigm is that when subjects fixated on the black lines, the red lines were located somewhat in the periphery of the retina, which may have contributed to the subjects' ability to ignore that material. Subjects, thus, may have used retinal acuity gradients to enhance selectivity. Finally, although numerous other attention paradigms have been developed for the visual modality (Gardner, 1973; Posner \& Boies, 1971; Shiffrin \& Gardner, 1972), for the most part, those paradigms have been used to address different questions, such as the level of information processing at which selective attention can operate.

One goal of the present research was to develop a visual analog to the auditory paradigms in which similar phenomena can be examined, and one in which the 
effect of retinal locus is minimized or eliminated. A task was developed in which a pair of digits was presented for a brief duration separated by about $5 \mathrm{deg}$ of visual angle with a word centered between the two digits. Subjects were asked to judge whether the two digits were of the same parity (both odd or both even) or of different parity and to ignore the centered word. Pilot research indicated that after the first few trials subjects were able to ignore the centered word, phenomenologically. With this task, subjects must be able to see both digits in order to respond correctly. The words, therefore, remain centered foveally, and any success in ignoring them must be accomplished centrally.

For initial comparison with phenomena observed in the auditory modality, we examined memory for the unattended words and the name effect. In most auditory attention experiments, subsequent memory for the unattended material is quite poor. If the present paradigm is conceptually similar to the auditory paradigms, then the subjects should be able to ignore the centered words and the memory for those words should be quite poor. The presence of a name effect would indicate, however, that some processing had taken place.

The present paradigm was used to address two questions concerning the fate of unattended information. The first question concerned the amount of processing capacity required to monitor unattended information. The name effect and related semantic effects described earlier imply that some processing of unattended material does occur. The present study examined whether that level of processing interferes with performance on the primary task. Some experiments have found that the mere presence of an unattended message has little effect on primary task performance (Moray \& O'Brien, 1967; Underwood \& Moray, 1971); other investigators have found a degradation in primary task performance (Keren, O'Hara, \& Skelton, 1977). In a paradigm similar to the one adopted here, Keren et al. had subjects perform a series of same-different tasks on pairs of separated letters. The tasks were adapted from Posner and Mitchell (1967). Centered between the two target letters, irrelevant letters were presented that were either consistent or inconsistent with the response required on that trial. They found that the consistency of the irrelevant material had a major influence on performance.

One problem with the Keren et al. (1977) paradigm for examining the processing demands of an unattended channel is verifying that their irrelevant material was, in fact, unattended. Instructing subjects that material is irrelevant does not insure that they will not attend to it. We found in pilot work that on a simple samedifferent judgment, subjects reported that they were able to make the required judgment and still attend to the centered word. This led us to choose a more difficult primary task for our actual experiments. A tendency to attend to the irrelevant material in the Keren et al. experiments might be further increased by the fact that the irrelevant material was drawn from the same set that the relevant material was drawn from.

We hoped to examine whether the presence of irrelevant material would affect primary task performance in a situation in which the irrelevant material was unrelated to the relevant material, in which there was evidence that the irrelevant material was not attended to (i.e., poor subsequent memory on a sensitive test and subjective reports), and, finally, in which there was evidence that the unattended irrelevant material was processed to some extent (i.e., a name effect). To test for the effect of unattended material, we used two groups, each with the same primary task (i.e., identifying whether two widely spaced digits were of the same or different parity). One group was presented a centered word on each trial that they were instructed to ignore. The other group received no centered words. If the mere presence of unattended material affects performance, it should show up as a change in reaction time and/or accuracy to the primary task.

The second question examined was the classic name effect (Moray, 1959). Usually, evidence for the name effect is documented as the percentage of subjects who report hearing their name or who follow instructions prefaced by their name. In addition to notice of the name, we expected to see a disruption in performance on the primary task on the trial on which the name was presented. This disruption might show up as a change in reaction time or a change in accuracy. We also presented each subject's name a number of times at spaced intervals to see if there was any change in the size of the name effect as a function of the number of repetitions.

\section{EXPERIMENT 1}

\section{Method}

Subjects. Fifty subjects participated in the experiment. They were enrolled in an introductory psychology course and received course credit for their participation. All subjects were naive to the purposes of the experiment.

Design. There were three groups in the experiment. Subjects in all three groups received six blocks of 20 trials each. On each trial, the stimulus consisted of two single-digit numbers separated by 5 deg of visual angle. The stimuli for group name (the primary condition) contained, in addition, a word on each trial centered between the two numbers. In each of Blocks 3-6, the subject's last name appeared once, as did a string of Xs the same length as the subject's last name. The task for the group name condition was to decide as rapidly as possible whether the two digits were of the same parity (both odd or both even) or of different parity. They were instructed to ignore the centered words. After the sixth block, the subjects were tested for recognition of the words.

Two control groups were employed to aid in the explication of possible outcomes in the name condition. The length group subjects received the same stimulus materials as the name group subjects. Their task, however, was to ignore the digits and to identify the length of the words as rapidly as possible. In a levels-of-processing sense, this would correspond to a low level of processing of the words (Craik \& Lockhart, 1972). Memory for 
the centered words following these length judgments was intended as a yardstick for measuring the memory for the centered words in the name group. The second control group (blank group) had the same primary task as the name group (i.e., identify the parity of the digits). No items, however, ever appeared between the digits and, of course, the blank group did not have a final recognition test.

There were 20 subjects in the name group, 20 in the blank group, and 10 in the length group.

Apparatus and Materials. Stimulus materials were presented on a Beehive Model 1 (CRT) computer terminal connected to the Dartmouth Time-Sharing System. The digits (and words, when involved) were presented on a single line in the middle of the screen. The bottom half of the screen was covered with an opaque mask that contained two white arrows positioned below the location where the digits appeared. A representation of the apparatus is shown in Figure 1. The entire stimulus line was presented simultaneously on each trial for a duration of $51 \mathrm{msec}$ The simultaneity was accomplished by printing the entire stimulus line below the opaque mask and then scrolling the line into view. With scrolling, the entire line is painted in under $1 \mathrm{msec}$. The digits were separated by 20 spaces $(5 \mathrm{~cm})$, and the subjects were seated at a viewing distance of $58 \mathrm{~cm}$ from the screen, making the visual separation of the digits $5 \mathrm{deg}$. The words in the name and length groups were centered between the pair of digits.

A black Plexiglas panel was placed over the keyboard of the computer terminal. The panel contained two central buttons positioned over the " $D$ " and " $K$ " keys. The keys were labeled "DIFF" and "SAME" in the name and blank groups and "SHORT" and "LONG" in the length group. There was also a row of five buttons in the upper left corner of the panel labeled " 1 " through " 5 ." These five buttons were used to record confidence ratings during the recognition test.

A Polytronics universal timer was placed between the terminal and the acoustic coupler. The timer allowed for the collection of real-time latencies to the nearest millisecond (Potts, 1976). The subjects' responses and latencies were transmitted directly to the timesharing system.

Only the digits $2,3,5$, and 8 were used as stimuli, since their visual confusability made the primary task more difficult. The digits were randomly paired for each subject on each trial, with the constraint that for half of the trials, the members of the pairs have the same parity and for the other half, they have different parities.

The same set of 20 words appeared in each of the six blocks. The words were chosen to vary in length from 3 to 10 letters. The wide variation in number of letters was used to span the

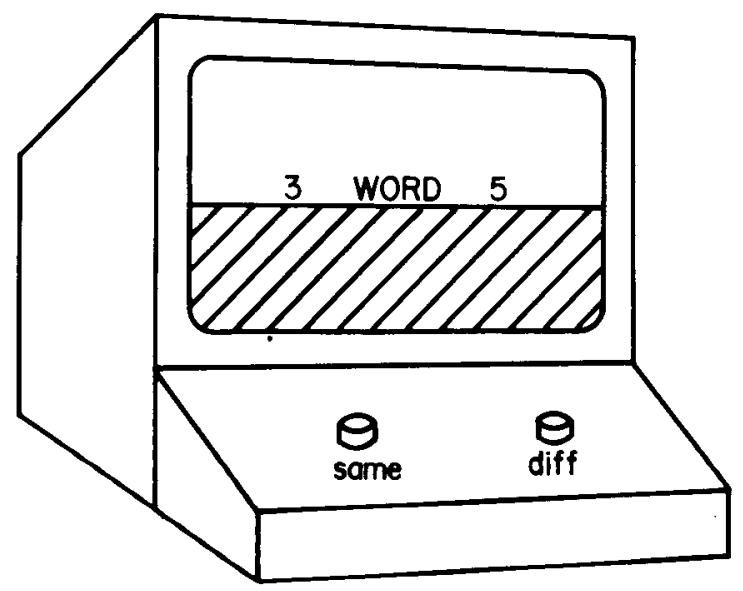

Figure 1. Display apparatus used in the two experiments. likely range of the length of subjects' last names. In that way, particularly long or short last names would not stand out from the other words on the basis of length. In fact, the last names of the subjects varied in length between 4 and 12 letters, with only one of the subjects' names being over 10 letters. All stimulus materials were presented in uppercase characters only. The list of 20 words is presented in the appendix.

For the name and length groups, the subject's last name was substituted once in each of the last four blocks. The position of the name within a block was chosen randomly from Trials 3.18 of that block. A string of Xs was also substituted for one of the words in each of the last four blocks. The string of Xs was matched in length with the subject's last name. The position of the string of Xs within a block was chosen in the same manner as the position of the name, subject to the further constraint that its position be at least three positions removed from the position of the name. Because of these substitutions, subjects saw each of the control words between four and six times during the course of the six blocks.

The recognition test for the name and length groups was a forced choice test in which each of the 20 centered words was paired with a single foil. The foils were matched overall with the target words on both length and frequency. The pairing of targets and foils was chosen at random for each subject, as was the ordering of the pairs. A string of Bs was used as the foil for the string of Xs, and another last name was used as the foil for the subject's last name.

Procedure. As subjects arrived, they were seated in front of the computer terminal and were read a set of instructions appropriate to their randomly determined group assignments. The name group was instructed that a pair of digits would appear very briefly above the arrows on the mask and that their task was to respond by pressing the "SAME" key if both numbers were odd or if both numbers were even and to respond by pressing the "DIFF" key if one of the numbers was odd and the other was even. They were to respond as rapidly as possible while trying to be accurate. The subjects were instructed to ignore the centered words, as we were interested in their ability to make rapid decisions in the presence of distraction (i.e., the words). No indication was given of the impending recognition test. The blank group was given similar instructions, except that no mention was made of the words or of distraction. The length group was instructed that a word would appear very briefly on the screen, centered between two digits. Their task was to press the key labeled "LONG" if there were six or more letters in the word and the key labeled "SHORT" if there were five or fewer letters. They were to respond as rapidly as possible while trying to be accurate.

Following the instructions, the timer was activated and the experiment began. Subjects were given feedback at the end of each block of 20 trials on percentage correct and mean latency for that block. During a block, the intertrial interval varied randomly due to the nature of the timesharing system, but a warning bell was sounded on the terminal $500 \mathrm{msec}$ prior to the start of each trial.

Following the sixth block, subjects in the name and length groups were informed that we were, in fact, interested in their memory for the words that had appeared during the experiment. Accordingly, they were then presented pairs of words side by side on the screen, one pair at a time, and were informed that one of the two words had been presented during the preceding blocks. They were to press the right key if the correct word was on the right and the left key if the correct word was on the left. They were then instructed to indicate their confidence by pressing one of the keys numbered 1-5, where 1 indicated a guess and 5 indicated certainty. The pair of words stayed in view until the subject indicated his choice and confidence. There was a total of 22 recognition trials with the 20 control words, the name, and the Xs. An experimental session lasted approximately $45 \mathrm{~min}$. 


\section{Results}

There were several aspects to the results of this experiment: performance on the primary task as a function of the presence or absence of distraction, memory for the unattended material on the recognition test, and an examination of performance on the primary task as a function of the nature of the centered item.

Latencies to the primary task as a function of block and group are displayed in Figure 2. Latencies over $4 \mathrm{sec}$ were discarded, and latencies on correct and incorrect trials were averaged together. The trials with the name or the Xs as the centered word were deleted from the name group. An analysis of variance was carried out, with blocks and groups (name and blank) as factors. The length group was not included, as they had a different primary task and were not directly comparable. Subjects in the length group were substantially faster and more accurate, as they appeared to have the easier task. The analysis of variance revealed a strong effect of blocks $[F(5,190)=33.86, p<.0001]$. There was absolutely no effect of group $[F(1,38)=.05$, $p=.81]$. In other words, the presence of a distractor did not impair performance on the primary task. The interaction did not approach significance.

The above analysis was conducted using correct and incorrect latencies pooled together, since it was felt that any interference produced by the distracting items might show up as an increase in reaction time, independent of correctness. As a check, we performed a similar analysis of variance in the more traditional fashion, using only correct latencies, and obtained the same pattern of results.

A separate analysis of variance was carried out using the same factors but using accuracy as the dependent measure (i.e., the percentage correct for each subject as a function of block and group assignment). These results are depicted in Figure 3. As can be seen, there was a general improvement in accuracy over blocks $[F(5,190)$ $=6.65, \mathrm{p}<.0001]$. Although the name group per. formed slightly better than the blank group on all blocks, the effect was not significant $[F(1,38)=2.96$, $\mathrm{p}=.09]$. The interaction did not approach significance.

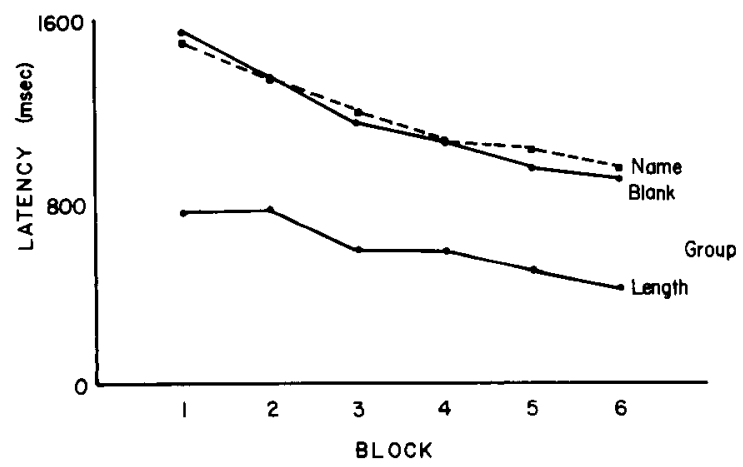

Figure 2. Latency as a function of block and group in Experiment 1 (limited to control trials for the name and length groups).

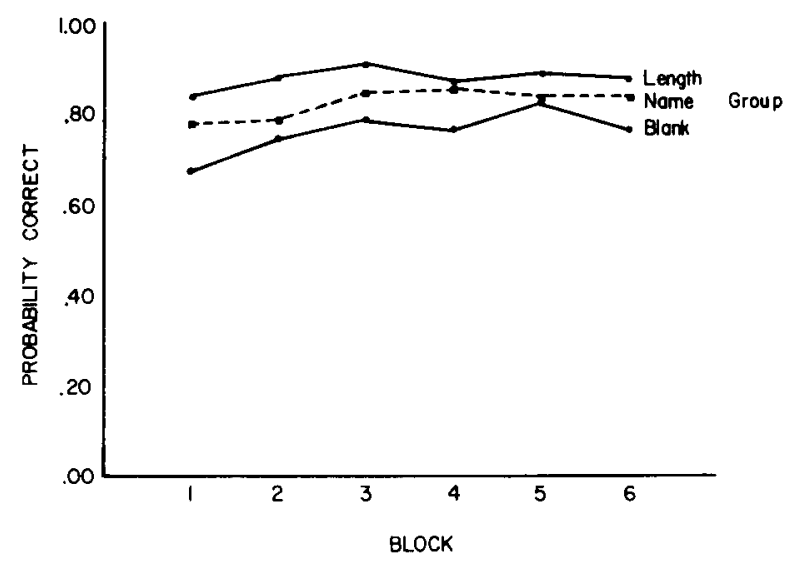

Figure 3. Probability correct as a function of block and group in Experiment 1 (limited to control trials for the name and length groups).

Memory for the centered words is presented in Table 1, along with subjects' confidence ratings of their choices. Performance on the control words was significantly higher than the chance level of $50 \%[t(19)=$ $10.67, p<.0001]$. Memory for the words in the name group, however, was substantially less than that in the length group $[t(28)=8.68, p<.0001]$. All but three of the subjects in the length group had perfect recognition scores. Recognition of the name was significantly above chance, using a binomial test $(\mathrm{p}=.006)$. Recognition of the Xs, however, did not differ from chance $(\mathrm{p}=.252)$. On the control words, the subjects were more confident when they were correct ( 3.05 vs. 2.23 ) than when they were incorrect $[t(19)=3.20, p=.0047]$. Subjects who recognized their names were quite certain of the fact, as indicated by their high confidence ratings.

The effect of the various item types on primary task latency is indicated in Table 2 . In addition to looking at the direct effect of the presence of the name and Xs, we also looked at the immediately following trial, on the possibility that the interference might extend to subsequent trials. An analysis of variance with blocks (only Blocks 3-6) and item type as factors was carried out on these latencies. The main effect of blocks was significant $[F(3,57)=3.68, p=.017]$. The effect of item type was also significant $[\mathrm{F}(4,76)=3.09, \mathrm{p}=.02]$, but the interaction was not significant $[F(12,228)=$ $1.57, \mathrm{p}>.10]$. A series of planned comparisons revealed that only the name trials differed significantly from the control trials.

Accuracy as a function of item type is shown in Table 3. An analysis of variance was performed with accuracy as the dependent measure and item type as the only factor. With the exception of the control items, a subject saw each of the item types only once per block, making it impossible to include block as a factor. We therefore collapsed across blocks. The effect of item type was not significant $[\mathrm{F}(4,76)<1]$. The small number of possible values for the dependent measure 
Table 1

Memory for the Centered Words in Experiment 1

\begin{tabular}{|c|c|c|c|c|c|c|}
\hline & \multicolumn{6}{|c|}{ Item Type } \\
\hline & \multicolumn{3}{|c|}{ Name Group } & \multicolumn{3}{|c|}{ Length Group } \\
\hline & Control & Name & $\mathrm{XXX}$ & Control & Name & $\mathrm{xXX}$ \\
\hline Percent Correct & 68 & 80 & 60 & 98 & 100 & 100 \\
\hline Mean Confidence of Corrects & 3.05 & 4.75 & 4.08 & 4.81 & 5.00 & 5.00 \\
\hline Mean Confidence of Errors & 2.23 & $1.00^{*}$ & $2.00^{*}$ & $3.25^{*}$ & & \\
\hline
\end{tabular}

*Based on relatively few observations.

Table 2

Latencies (in Milliseconds) as a Function of Item Type and Block in Experiment 1

\begin{tabular}{lcrrrrr}
\hline \multirow{2}{*}{$\begin{array}{c}\text { Item } \\
\text { Type }\end{array}$} & 1 & 2 & 3 & 4 & 5 & \multicolumn{1}{c}{ Block } \\
\hline Control & 1500 & 1350 & 1199 & 1072 & 1034 & 949 \\
Name & & & 1374 & 1224 & 1403 & 942 \\
Name +1 & & & 1133 & 1132 & 1033 & 1121 \\
XXx & & & 1251 & 1307 & 1005 & 1009 \\
Xxx +1 & & & 1071 & 966 & 938 & 907 \\
\hline
\end{tabular}

makes this analysis of variance somewhat questionable, but the observed percentages, along with the very low $\mathrm{F}$, probably do indicate that item type did not influence primary task accuracy.

\section{Discussion}

The visual paradigm utilized in this experiment was successful in obtaining many of the effects obtained with auditory paradigms. Phenomenologically, the task appears similar to the traditional paradigms. All subjects in the name group reported that the centered words appeared to disappear after the first several trials.

As expected, the recognition memory for the centered words was quite low, although significantly above chance. To get a sense of just how good the memory for the unattended words was, we used as a comparison the recognition scores from the length group. The subjects in the length group had to identify the length of the centered words as rapidly as possible. Even though this would generally be considered a low level of processing and the words were only exposed for $51 \mathrm{msec}$, subjects were virtually perfect on the subsequent recognition test and far superior to the subjects in the name group (99\% correct for the length group vs. $68 \%$ for the name group). The $68 \%$ is especially low, considering that the control words were presented an average of six times each. Inspection of the data revealed that the greater than chance memory of the subjects in the name group was probably the result of a failure to ignore the centered words during the first several trials. Experiment 2 was designed to test this possibility.

A fairly robust name effect was also obtained in this experiment. When asked on the recognition test, $80 \%$ of the subjects in the name group reported that they had seen their name during the experiment, and they almost
Table 3

Percent Correct as a Function of Item Type and Block in Experiment 1

\begin{tabular}{lcccccc}
\hline & \multicolumn{6}{c}{ Item } \\
\cline { 2 - 7 } \multicolumn{1}{c}{ Type } & 1 & 2 & 3 & 4 & 5 & 6 \\
\hline Control & 78 & 79 & 85 & 87 & 84 & 84 \\
Name & & & 97 & 80 & 80 & 95 \\
Name +1 & & & 70 & 75 & 80 & 95 \\
XXX & & & 85 & 75 & 85 & 90 \\
XXX +1 & & & 90 & 70 & 90 & 90 \\
\hline
\end{tabular}

invariably checked the highest possible confidence rating. The presentation of the name also produced a significant disruption on the primary task, resulting in longer reaction times on those trials. Although there appeared to be a large and stable disruption on the first three repetitions of the name (see Table 2), inspection of the individual data indicated that most subjects had only one large disruption, ranging from 300 to $1,500 \mathrm{msec}$, and that different subjects exhibited the disruption in different blocks.

Moray (1959) presented each subjects' name three times on the unattended ear, with the last occurrence forewarned. On each of the first two presentations, $33 \%$ of the subjects reported hearing their name. While this, at first, may seem discrepant with our $80 \%$ figure, the discrepancy may result largely from the measures used. If we assume that $33 \%$ of the subjects hear their name on each presentation and that the different presentations constitute independent events, then the probability of hearing one's name at least once in four presentations would be $1-.67^{4}$, or $80.2 \%$. This value compares favorably with our obtained value and argues further for the similarity between the paradigms.

Many subjects gave further evidence of the name effect through overt displays during the experiment. These displays included exclamations, laughter, and various comments expressing surprise. One problem in studying the name effect is that due to the surprise quality of the effect, the name can be presented only a few times to each subject without making him suspect the nature of the experiment. Latencies are often quite variable, so that when only a few observations are available, robust effects are required to produce significant differences.

A major concern of the experiment was to see if the 
mere presence of an unattended channel affects performance on the primary task. The outcome was fairly clear. The presence of an unattended channel did not interfere with performance on the primary task. The latencies were nearly identical for the two relevant groups, and accuracy, although it produced no significant differences, slightly favored the name group over the blank group.

The necessary requirements described in the introduction for testing the above question were met in Experiment 1 . The poor memory for the centered words on a sensitive test showed that the words were not attended to, whereas the presence of the name effect indicated that some processing of the words did take place. In addition, the test of the processing resources question did not suffer from the same paucity of observations that may have influenced the exploration of the name effect. Each of the 20 subjects in each of the two groups contributed 96 observations to the comparison.

Thus, even though the subjects process the unattended information to some level, possibly semantic, that processing does not appear to detract from performance on the primary task. Experiments such as Keren et al. (1977) may find the opposite effect because their subjects, on occasion, attend to the irrelevant material.

In light of the preceding claim that the monitoring of the unattended channel requires no measurable processing capacity, why does the presence of one's name on the unattended channel lead to a measurable disruption? Other studies showing semantic processing of unattended material (e.g., Lackner \& Garrett, 1972) report no awareness or later memory for the unattended information on the part of the subjects. We assume that the name is initially processed to the same extent as other unattended information. This amount of processing for the name, however, is sufficient to lead either to awareness or to further processing. This assumption is consistent with the notion that the name has a lower awareness threshold than other words (Howarth \& Ellis, 1961). The awareness of one's name or the additional processing would then be the cause of the disruption to the primary task.

\section{EXPERIMENT 2}

Experiment 2 was designed to explore the possibility that the greater than chance recognition of the control words in Experiment 1 was due to failures of attention during the early trials. The experiment used a single group of subjects, similar to the name group of the preceding experiment, except that a new group of control words was introduced in the final block. The newly introduced control words were, then, tested on the subsequent recognition memory test. The rationale for Experiment 2 was that failures to ignore the centered words during the early trials should not affect memory for words presented only in the final block.

\section{Method}

Twenty new subjects were drawn from the same population used in Experiment 1. The apparatus and procedure were identical to those used in Experiment 1. The stimulus materials were also the same, except that a new set of 20 control words was introduced into the sixth (final) block. These words are listed in the appendix. Only the new control words were tested on the subsequent recognition test, and the same incorrect alternatives were used as in Experiment 1. As in the first experiment, the subject's last name and a string of Xs each replaced a control word in Blocks $3-6$. Therefore, any one subject saw only 18 of the 20 new control words. During the recognition test, subjects were tested only on the new control words that were actually presented.

\section{Results}

The results of primary concern in this experiment are the performance scores on the final recognition test. These results are shown in Table 4 . The probability correct on the new control words was not significantly different from chance $(52 \%$ vs. $50 \%)[\mathrm{t}(19)=.511$, $p=.621]$. As in Experiment 1, the probability of subjects' recognizing their name was above chance using a binomial test $(\mathrm{p}=.012)$. Unlike Experiment 1, subjects recognized the Xs at an above-chance rate $(p=.002)$. Even though subjects were at or near chance on the forced recognition test, their confidence ratings indicated that they may have had some memory for the unattended words. Confidence ratings on correct recognition trials were significantly higher $(2.75$ vs. 2.36$)$ than those on incorrect trials $[t(19)=2.43, p=.024]$. This confidence split, however, was far smaller in magnitude than the split found in Experiment 1.

Performance on the primary task was similar to that found for the name group in the first experiment. The effect of the various item types on primary task latency is shown in Table 5. An analysis of variance with blocks (Blocks 3-6 only) and item type as factors was carried

Table 4

Memory for the Centered Words in Experiment 2

\begin{tabular}{lrcc}
\hline & \multicolumn{3}{c}{ Item Type } \\
\cline { 2 - 4 } & Control & Name & xxx \\
\hline Percent Correct & 52 & 80 & 85 \\
Mean Confidence of Corrects & 2.75 & 4.56 & 4.24 \\
Mean Confidence of Errors & 2.36 & $2.50^{*}$ & $1.00^{*}$ \\
\hline
\end{tabular}

*Based on relatively few observations.

Table 5

Latencies (in Milliseconds) as a Function of Item Type and Block in Experiment 2

\begin{tabular}{lcccccc}
\hline & \multicolumn{6}{c}{ Item } \\
\cline { 2 - 7 } \multicolumn{1}{c}{ Type } & 1 & 2 & 3 & 4 & 5 & 6 \\
\hline Control & 1485 & 1295 & 1130 & 1080 & 1047 & 1055 \\
Name & & & 1443 & 1492 & 1274 & 1161 \\
Name +1 & & & 1209 & 1140 & 1023 & 1151 \\
xxx & & & 1481 & 1288 & 1066 & 1120 \\
Xxx +1 & & & 1310 & 1138 & 1077 & 1087 \\
\hline
\end{tabular}


Table 6

Percent Correct as a Function of Item Type and Block in Experiment 2

\begin{tabular}{lcrrrrr}
\hline & \multicolumn{6}{c}{ Item } \\
\cline { 2 - 7 } \multicolumn{1}{c}{ Type } & 1 & 2 & 3 & 4 & \multicolumn{1}{c}{5} & \multicolumn{1}{c}{6} \\
\hline Control & 80 & 89 & 88 & 89 & 90 & 88 \\
Name & & & 90 & 95 & 100 & 86 \\
Name +1 & & & 90 & 90 & 67 & 95 \\
xxx & & & 86 & 100 & 90 & 81 \\
xxx +1 & & & 86 & 95 & 86 & 90 \\
\hline
\end{tabular}

out on the latency data. The main effect of item type was significant $[F(4,76)=3.52, p=.0109]$. Planned comparisons revealed that both the name and the $\mathrm{X}$ trials were significantly slower than the control trials. The main effect of block was not significant, although the mean latencies did show a general decrease across Blocks 3-6, as in Experiment 1. The interaction did not approach significance.

Accuracy as a function of block and item type is shown in Table 6. As in the previous experiment, an analysis was carried out on the effect of item type averaged across the last four blocks. The effect of item type did not approach significance $[F(4,76)<1]$.

\section{Discussion}

The results from the second experiment indicate that memory for the unattended words is, at best, very weak. The significant confidence rating split may imply that the confidence ratings are more sensitive than the forced choice decision. The recognition scores lend credence to the suggestion that the greater than chance recognition scores in the first experiment may have been due primarily to failures of attention during the early trials.

As in the first experiment, there was a robust name effect, as evidenced by both a significant disruption during the primary task and high recognition scores. The $\mathrm{Xs}$ were more disruptive and better recognized than in the first experiment.

The results from the two experiments strongly support the claim that the paradigm used represents a viable visual analog to standard auditory attention paradigms.

\section{REFERENCE NOTE}

1. Neisser, U. Selective reading: A method for the study of visual attention. Paper presented at the Nineteenth International Congress of Psychology, London, 1969.

\section{REFERENCES}

Broadent, D. E. Perception and communication. London: Pergamon, 1958

Craik, F. I. M., \& Lockhart, R. S. Levels of processing: A framework for memory research. Journal of Verbal Learning and Verbal Behavior, 1972, 11, 671-684.

GaRDNER, G. T. Evidence for independent parallel channels in tachistoscopic perception. Cognitive Psychology, 1973, 4, 130-155.
Howarth, L., \& Ellis, K. The relative intelligibility threshold for one's own and other people's names. Quarterly Journal of Experimental Psychology, 1961, 13, 236-240.

Kahneman, D. Attention and effort. Englewood Cliffs, N.J: Prentice-Hall, 1973.

Keren, G., O'Hara, W. P., \& Skelton, J. M. Level of noise processing and attentional control. Journal of Experimental Psychology: Human Perception and Performance, 1977, 3, 653-664.

Lackner, J. R., \& Garrett, M. F. Resolving ambiguity: Effects of biasing context in the unattended ear. Cognition, 1972, 1 , 359-372.

LEWIS, J. L. Semantic processing of unattended messages using dichotic listening. Journal of Experimental Psychology, 1970, 85, 225-228.

MACKAY, D. G. Aspects of the theory of comprehension, memory and attention. Quarterly Journal of Experimental Psychology, $1973,25,22-40$.

Moray, N. Attention in dichotic listening: Affective cues and the influence of instructions. Quarterly Journal of Experimental Psychology, 1959, 11, 56-60.

Moray, N., \& O'Brien, T. Signal detection theory applied to selective listening. Journal of the Acoustical Society of America, $1967,42,765-772$.

Newstead, S. E., \& Dennis, I. Lexical and grammatical processing of unshadowed messages: A reexamination of the MacKay effect. Quarterly Journal of Experimental Psychology, $1979,31,477-488$

Posner, M., \& Boles, S. J. Components of attention. Psychological Review, 1971, 78, 391-408.

Posner, M., \& Mitchell, R. F. Chronometric analysis of classification. Psychological Review, 1967, 74, 392-409.

PotTs, G. R. Use of a campus-wide timesharing computer to run reaction time experiments. Behavior Research Methods \& Instrumentation, 1976, 8, 179-181.

Shiffrin, R. M., \& Gardner, G. T. Visual processing capacity and attentional control. Journal of Experimental Psychology, $1972,93,72-82$.

Treisman, A. M. Contextual cues in selective listening. Quarterly Journal of Experimental Psychology, 1960, 12, 242-248.

Treisman, A. M. Monitoring and storage of irrelevant messages in selective attention. Journal of Verbal Learning and Verbal Behavior, 1964, 3, 449-459.

Treisman, A. M., Squire, R., \& Green, J. Semantic processing in dichotic listening? A replication. Memory \& Cognition, 1974, 2, 641-646.

Underwood, G., \& Moray, N. Shadowing and monitoring for selective attention. Quarterly Journal of Experimental Psychology, 1971, 23, 284-296.

\section{APPENDIX}

Control Words (Experiment 1)

BUILDING OCEAN SHEET WALL GLASS KEY PIPE HANDLE SHOE SNEAKER SPARK INSECT COLUMN FINGER FLASHLIGHT TEST WINDOW WELL BOOK SIDEWALK

Foils (Experiments 1 and 2) SMOKE TODAY TREE LETTER DOOR BICYCLE STICK SCREWDRIVER POST CURTAIN LINE CROSS PENCIL HAIR OIL BRANCH CHALK SCHOOL STREET BELT

\section{New Control Words (Block 6-Experiment 2)}

BRUSH SQUARE ROW TELEPHONE TABLE LOBSTER WATER TRAIN ANIMAL BALL CLIFF JACKET CAR CIGARETTE ICE TRUCK RECORD CORNER FISH STAIRS

(Received for publication November 13; 1979; revision accepted July 3,1980 .) 